\title{
Hamiltonian assignment for open quantum systems
}

\author{
Eugene F. Dumitrescu $\odot^{*}$ and Pavel Lougovski $\odot^{\dagger}$ \\ Quantum Information Science Group, Computational Sciences and Engineering Division, \\ Oak Ridge National Laboratory, Oak Ridge, Tennessee 37831, USA
}

(Received 13 January 2020; accepted 10 July 2020; published 14 August 2020)

\begin{abstract}
We investigate the problem of determining the Hamiltonian of a locally interacting open quantum system. To do so, we construct Hamiltonian estimators based on inverting a set of stationary, or dynamical, HeisenbergLangevin equations of motion which rely on a polynomial number of measurements and model parameters. To validate our Hamiltonian assignment methods we numerically simulate one-dimensional $X X$-interacting spin chains coupled to thermal reservoirs. We provide general bounds on the scalability and assignment error in the presence of noise. In addition to discussing some details of practical implementations we find that, in a dynamical setting, the Hamiltonian estimator's accuracy increases when relaxing the environment's physicality constraints.
\end{abstract}

DOI: 10.1103/PhysRevResearch.2.033251

\section{INTRODUCTION}

Fault tolerant quantum computation provides a framework for digitally decomposing unitary operators using a polynomial number of one- and two-qubit operations drawn from a universal gate set [1]. For noisy intermediate scale quantum (NISQ) hardware, characterized by fixed gate fidelities and limited coherence times, digitizing a quantum simulation unitary is too costly in terms of the polynomial scaling circuit depth.

However, if a programmable quantum device's many-body dynamics are described by an underlying Hamiltonian $H$, it is prudent to consider digital-analog decompositions [2] leveraging $H$. It has been proposed that, in such a case, the target unitary can be decomposed as a sequence of native analog unitaries $U=\exp (-i H t)$ interleaved with programmable singlequbit operations. For certain applications, such as many-body simulations [3], the gate complexity, quantified by the total number of applications of the many-body evolution operator $U$ and local rotations, may be significantly smaller than that of the digitized decomposition.

The digital-analog quantum simulation's error must be bounded, e.g., in terms of the distance between the target and digital-analog unitaries, in order to certify an accurate simulation. It therefore follows that, in order to upper bound the simulation error, one must first precisely characterize the Hamiltonian generating the many-body operation.

While some prior results regarding Hamiltonian estimation exist (e.g., process tomography [4] and Bayesian Hamiltonian

\footnotetext{
*dumitrescuef@ornl.gov

†lougovskip@ornl.gov

Published by the American Physical Society under the terms of the Creative Commons Attribution 4.0 International license. Further distribution of this work must maintain attribution to the author(s) and the published article's title, journal citation, and DOI.
}

learning [5]), for scalable methods (local Hamiltonian tomography [6]) the estimation task is complicated by interactions coupling the principle system of interest to unwanted environmental degrees of freedom. To address this outstanding issue, we study the problem of assigning a Hamiltonian to an open quantum system, provided the principle system and environmental interactions are both geometrically local.

We first formulate the task of Hamiltonian inference and summarize previous results. Afterwards we generalize the Hamiltonian learning protocols to the context of open quantum systems and perform numerical simulations in order to validate and analyze our techniques in two distinct noisy settings. We conclude by discussing generalizations, practical constraints, and future directions for Hamiltonian learning.

\section{BACKGROUND}

Hamiltonian tomography refers to the task of estimating a Hamiltonian $H$ given access to states evolving under $H$. While this task is exponentially costly in general, the tomography of local Hamiltonians has recently attracted significant attention [6-9] due to its scalability. We are interested in determining $k$-local Hamiltonians of the form

$$
H=\sum_{i} c_{i} S_{i},
$$

where each $S_{i}$ is an operator supported on $k$ spatially connected sites. We work in the Pauli basis, such that all $k$ local operators can be written as $S_{i}=\bigotimes_{j=0}^{k-1} \sigma_{j}^{\mu_{j}}$ where $\mu_{j} \in$ $\{I, X, Y, Z\}$, where the index $j$ runs over spatially connected sites.

Suppose we have access to either (i) eigenstates $\left|\psi_{n}\right\rangle$ of $H$, with $H\left|\psi_{n}\right\rangle=E_{n}\left|\psi_{n}\right\rangle$ or (ii) thermal states $\rho=\exp (-\beta H) / \mathcal{Z}$ where $\mathcal{Z}=\operatorname{Tr}[\exp (-\beta H)]$ is the partition function. Aside from the trivial phase factors, both states are stationary under Hamiltonian dynamics. In the Heisenberg picture expectation values taken with respect to these states are likewise stationary 
and we may write

$$
\langle\dot{O}\rangle=\frac{-i}{\hbar}\langle[O, H]\rangle=0
$$

for any observable $O$. Inserting Eq. (1), selecting an input operator basis $\left\{O_{j}\right\}$, and measuring the commutators $\left\langle\left[O_{j}, H\right]\right\rangle$, we may express the set of linear equations $\sum_{j, i}\left\langle\left[O_{j}, S_{i}\right]\right\rangle c_{i}=$ 0 concisely in matrix form as

$$
A \vec{c}=0,
$$

where we have introduced the matrix $A$ with elements $A_{i, j}=\left\langle\left[O_{j}, S_{i}\right]\right\rangle$ and the Hamiltonian coefficient vector $\vec{c}=$ $\left(c_{1}, \ldots, c_{n}\right)^{T}$. Note that in principle $A$ need not be a square matrix as its dimensions are determined by the number of accessible correlation measurements. Since the operators $S_{i}$ are $k$-local and we have the freedom to choose $O_{j}$ from a local basis, most correlators will vanish, due to spatially nonoverlapping $\left(O_{j}, S_{i}\right)$ pairs, and $A$ will be sparse.

In practice, entries of $A$ arise from noisy measurements which may lead to an erroneous evaluation of eigenvalues. To improve the numerical stability of the inversion problem, one could reformulate the problem as a convex optimization problem, minimize $\|A \vec{c}\|_{2}^{2}$, which is equivalent to maximizing a Gaussian $\log$ likelihood, maximize $\log \mathrm{e}^{-\vec{c}^{T} A^{T} A \vec{c}}$. The latter formulation is convenient for incorporating Bayesian uncertainty quantification methods, e.g., to treat noise in the matrix $A$. If $A$ is a square matrix, then $A \vec{c}=0$ has a unique solution only if $A$ has a nondegenerate zero eigenvalue. Note that Eq. (3) holds for all Hamiltonians $a H$ defined up to the scalar factor $a$. In order to avoid trivial solutions we may reformulate assignment into the constrained optimization task: minimize $\|A \vec{c}\|_{2}^{2}$, subject to $\|\vec{c}\|_{2}^{2}=1$. The solution is then the row vector of $V^{T}$ associated with the minimal singular value in $A$ 's singular value decomposition $A=U \Sigma V^{T}$. We return to the issue of numerical stability when considering noise below.

While the homogeneous operator equations derived from steady states provide a simple formalism, preparing eigenstates and thermal states of an unknown Hamiltonian may be challenging or time consuming. Earlier work has therefore also explored Hamiltonian estimation in a dynamical context $[7,8]$. In the Heisenberg picture an observable $O$ evolves as $O(t)=e^{i t H} O(0) e^{-i t H}$, where $O(0)$ denotes the observable at time $t=0$ where it coincides with its Schrödinger picture counterpart. Integrating Eq. (2) over an infinitesimal time $\delta t$, we write

$$
\left\langle O_{i}(\delta t)\right\rangle_{j}-\left\langle O_{i}(0)\right\rangle_{j}=-i \delta t\left\langle\left[O_{i}(0), H\right]\right\rangle_{j}+\mathcal{O}\left(\delta t^{2}\right),
$$

where the trace is taken with respect to an initial state $\rho_{j}$, which serves as an input degree of freedom. Considering the small time evolution of a set of operators $O_{i}$, with respect to a set of initial states $\rho_{j}$, we consider the set of heterogeneous Heisenberg equations of motion defined by the measurement settings' vector $W_{i j}=\left\langle O_{i}(\delta t)\right\rangle_{j}-\left\langle O_{i}(0)\right\rangle_{j}$ and matrix elements $A_{i, j, l}^{\prime}=\delta t c_{l}\left\langle\left[S_{l}, O_{i}\right]\right\rangle_{j}$. This can be expressed as

$$
A^{\prime} \vec{c}=\vec{W},
$$

and, as before, the assigned Hamiltonian will correspond to the solution vector $\vec{c}$ optimizing $\min _{\vec{c}}\left\|A^{\prime} \vec{c}-\vec{W}\right\|_{2}^{2}$.

\section{OPEN SYSTEM GENERALIZATION}

Unfortunately, the inability to evolve by purely unitary dynamics limits the applicability of closed Hamiltonian learning. Realistic quantum systems are open and, in the presence of unknown environmental interactions, evolve dissipatively. In order to incorporate environmental couplings in our framework, we consider a Markovian master equation dynamics for a density operator $\partial_{t} \rho=\mathcal{L}[\rho]$ generated by the quantum Liouvillian $\mathcal{L}$. Specifically, we consider a Lindblad equation given by $\mathcal{L}[\rho]=\frac{-i}{\hbar}[H, \rho]+\mathcal{D}[\rho]$, where $\mathcal{D}=\sum_{n, m} \gamma_{n m}\left(L_{n} \rho L_{m}^{\dagger}-\frac{1}{2}\left\{L_{m}^{\dagger} L_{n}, \rho\right\}\right)$. Motivated by locality, we consider the $\left\{L_{n}\right\}$ operators to form an orthonormal basis spanning the manifold of $J$-local superoperators. The coefficient matrix $\gamma$ is constrained to be positive semidefinite in order to represent a physical map between positive semidefinite density operators [10]. An observable's dynamics will now be given by the Heisenberg-Langevin master equation $\dot{O}=\frac{-i}{\hbar}[O, H]+\mathcal{D}^{\dagger}[O]$, where $\mathcal{D}^{\dagger}[O]=$ $\sum_{n, m} \mathcal{D}_{n, m}^{\dagger}[O]=\sum_{n, m} \gamma_{n m}\left(L_{m}^{\dagger} O L_{n}-\frac{1}{2}\left\{L_{m}^{\dagger} L_{n}, O\right\}\right)$. As in previous sections, a model for the equations of motion is learnable with polynomial resources if one imposes a locality constraint on the geometric locality of the Lindbladian dissipators. In the discussion below we assume that dissipative correlations are bounded by a length scale $J$, such that we speak of $K$-local Hamiltonians and $J$-local dissipators.

Lindbladian fixed points, satisfying $\mathcal{L}[\rho]=0$, generalize the notion of Hamiltonian steady states and their stationary dynamics can be used to generalize Eq. (3). That is, consider the linear equations $A \vec{c}+B \vec{\gamma}=0$, where, in addition to $A$, $B$ is defined by its elements $B_{i}^{n, m}=\left\langle L_{n}^{\dagger} O_{i} L_{m}-\frac{1}{2}\left\{L_{n}^{\dagger} L_{m}, O_{i}\right\}\right\rangle$. As before, the set of linear equations can be invoked as $C \vec{x}=0$, where $C=(A \mid B)$ acts on a composite HamiltonianLindbladian model space spanned by the configuration vector $\vec{x}=\left(\vec{c}^{T}, \vec{\gamma}^{T}\right)^{T}$. While Lindbladian learning has been recently investigated [11], where it was shown that reconstructing strongly dephasing jump operators is difficult under certain conditions, our focus is on precisely estimating the Hamiltonian component, possibly at the expense of the environmental sector.

Let us also consider finite-time evolution which is applicable if Lindbladian fixed points are unavailable. As before, the linearized Heisenberg-Langevin evolution can be constructed at the cost of an approximation error $\delta t^{2}$. With the input state degree of freedom $\rho_{j}$, the matrix elements may be defined as $B_{i, j}^{\prime n, m}=\delta_{t}\left\langle L_{n}^{\dagger} O_{i} L_{m}-\frac{1}{2}\left\{L_{n}^{\dagger} L_{m}, O_{i}\right\}\right\rangle_{j}$. The dynamical equations of motion are simply $C^{\prime} \vec{x}=\vec{W}$, where $C^{\prime}=\left(A^{\prime} \mid B^{\prime}\right), \vec{W}$, and $A^{\prime}$ have been defined in the closed systems context.

\section{MODEL SYSTEM}

We present numerical simulations in order to quantitatively analyze the behavior of the open system Hamiltonian learning methods outlined above. To do so, we consider onedimensional spin chains consisting of $N$ sites with a Hamiltonian $H=H_{0}+H_{I}$. Here, $H_{0}=\sum_{i} \vec{c}_{i} \cdot \vec{\sigma}_{i}$ consists of singlequbit interactions, with $\vec{\sigma}_{i}=\left(\sigma_{i}^{x}, \sigma_{i}^{y}, \sigma_{i}^{z}\right)$, and the nearestneighbor interactions are given by $H_{I}=\sum_{i} J_{i, i+1} \sigma_{i}^{x} \sigma_{i+1}^{x}$. We simulate a system with Hamiltonian interactions 
$\vec{c}=(0.5,0,-2.55), J_{i, i+1}=0.25$ at each site. The system's translational symmetry could be used to reduce the size of the model space, but we do not expect this to hold in general and work with the unconstrained model, assigning distinct parameters to each region.

In order to mimic environmental effects each spin is then subjected to thermal noise described by thermal excitation and relaxation operators which are written as $L_{+}=\sqrt{g_{+}} \sigma^{+}$ and $L_{-}=\sqrt{g_{-}} \sigma^{-}$. Here, $g_{+}=g \bar{n} / 2, g_{-}=g(\bar{n}+1) / 2, \bar{n}$ is a thermal occupation number, and $g$ is the reservoir-spin coupling strength. An operator $O$ supported on a given site evolves dissipatively under $\mathcal{D}_{\bar{n}}^{\dagger}[O]=\frac{g_{-}}{4}\left(\sigma^{+} O \sigma_{-}-\right.$ $\left.\left\{\sigma^{-} \sigma^{+}, O\right\} / 2\right)+\frac{g_{+}}{4}\left(\sigma^{-} O \sigma^{+}-\left\{\sigma^{+} \sigma^{-}, O\right\} / 2\right)$. Expanding the ladder operators $\sigma^{ \pm}=(X \pm i Y) / 2$ and regrouping the terms we see that this expression may be rewritten in the Pauli basis as $\mathcal{D}_{\bar{n}}^{\dagger}=\mathcal{D}_{X, X}^{\dagger}[O]+\mathcal{D}_{Y, Y}^{\dagger}[O]+\mathcal{D}_{X, Y}^{\dagger}[O]+\mathcal{D}_{Y, X}^{\dagger}[O]$, where the coefficients are $\gamma_{X X}=\gamma_{Y Y}=\left(g_{+}^{2}+g_{-}^{2}\right) / 4$ and $\gamma_{X Y}=\gamma_{Y X}^{*}=i\left(g_{-}^{2}-g_{+}^{2}\right) / 4$.

\section{EQUILIBRIUM LEARNING}

In order to the simulate the steady state learning protocol, we first solve for the fixed-point density operator satisfying $\mathcal{L}[\rho]=0$, where $\mathcal{L}$ is defined above [12]. Next, we select the Pauli basis to express the Hamiltonian and Lindbladian model spaces and the set of input operators $\left\{O_{i}\right\}$. In practice one should begin with a small model space and increase its size until adequate convergence, but for simplicity we take as our candidate model the space of $K=2, J=1$-local configurations. In order to fully explore the maximal quality of learning model we take $\left\{O_{i}\right\}$ to be the overcomplete union of all 1-, 2-, 3-, and 4-local operators.

The top panel in Fig. 1 illustrates the fixed-point model estimation protocol for an $N=5$ site chain with open boundary conditions. The horizontal axis denotes the cardinality of the set $\left\{O_{i}\right\}$. We have swept through operators of each locality, as indicated by the dashed lines, which are enumerated from the left to the right ends of the chain. Plotted along the vertical axis are the Hamiltonian estimator errors $\Delta_{H}=$ $\left\|\vec{c}_{T}-\vec{c}_{E}\right\|_{2} /\left\|\vec{c}_{T}\right\|_{2}$, where $T, E$ refer to the true and estimator models and, recalling that $\vec{x}=\left(\vec{c}^{T}, \vec{\gamma}^{T}\right)^{T}$, the total estimator error $\Delta_{H-L}=\left\|\vec{x}_{T}-\vec{x}_{E}\right\|_{2} /\left\|\vec{x}_{T}\right\|_{2}$. The estimators are constructed by decomposing the correlation matrix $C=U \Sigma V^{T}$ and selecting as a solution the row vector of $V^{T}$ associated with the minimal singular value $s_{0}$ of $\Sigma=\operatorname{diag}\left(s_{m-1}, \ldots, s_{0}\right)$. In our numerical examples, given sufficient input data, the reconstruction errors in Fig. 2 are only limited by numerical round-off errors in the singular value decomposition of the correlation matrix.

In order to decompose the model space into the image and kernel of the correlation matrix the cardinality of the measurement set $O_{i}$ should exceed the size of the model space which generally scales as $\mathcal{O}\left(V\left(4^{K}+4^{2 J}\right)\right)$, the space of $K(J)$-local Hamiltonian (Lindbladian) operators can is expanded in a $4^{K(2 J)}$-dimensional basis of the Pauli operators $(I, X, Y, Z)^{\otimes K(2 J)}$, where $V=N, 3 N, N^{2}$ for a onedimensional (1D), ladder, and 2D geometry, respectively. For a collection of $d$-dimensional systems this bound generalizes to $\mathcal{O}\left(V\left(d^{2 K}+d^{4 J}\right)\right)$. Figure 1 illustrates this convergence, at the discontinuity, as the input $\left\{O_{i}\right\}$ space grows for a five-site
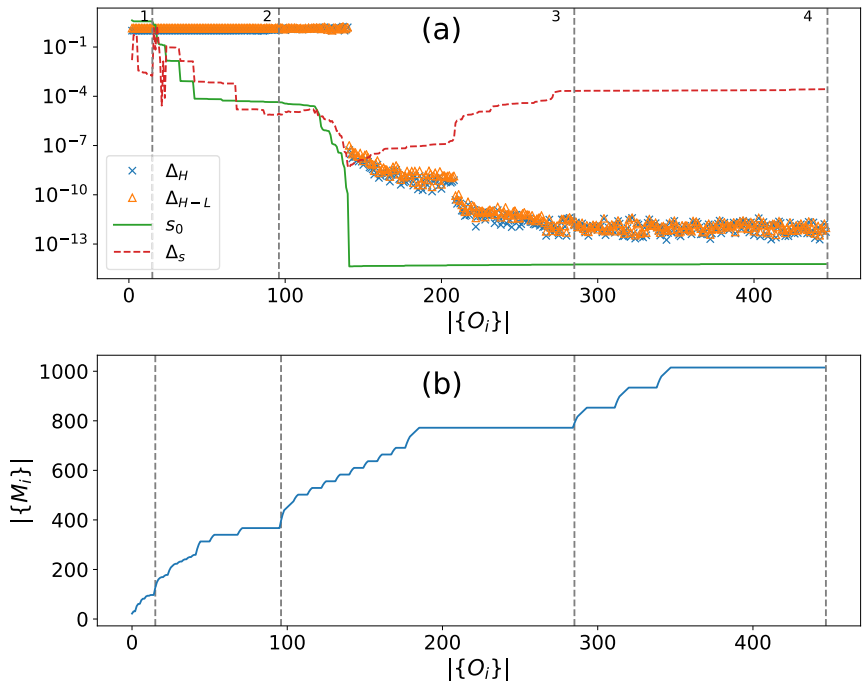

FIG. 1. Top panel: Steady state estimator error and low-lying singular values as a function of the input $\left\{O_{i}\right\}$ 's cardinality [i.e., the number of rows in Eq. (3)]. The gray dashed lines enumerate the transitions between input operator localities. The normalized Hamiltonian (Lindbladian) estimator error $\Delta_{H}\left(\Delta_{H-L}\right)$ is given by the blue $\times$ (orange triangle) markers while the minimal singular value $s_{0}$ and gap to the next singular value $\Delta_{s}$ are denoted by the green solid and red dashed lines respectively. Note the discontinuity in estimator error and singular spectrum upon the correlation matrix becoming left invertible (our five-site model contains 141 free parameters). Bottom panel: Measurement complexity $\left|\left\{M_{i}\right\}\right|$, which is generated as the union of unique operators contained in the matrix elements of $C$, in terms of the cardinality $\left|\left\{O_{i}\right\}\right|$. The local, $X X$, and thermal interaction parameters are $\vec{c}=(0.5,0,-2.55), J=0.25, g=0.05$, respectively.

model with scaling corrections due to finite-size effects. The minimal singular value $s_{0}$ likewise approaches zero, such that Eq. (3) is well approximated by the associated right singular vector. The solution's uniqueness is indicated by the large singular gap $\Delta_{s}=s_{1}-s_{0}$, in the sense that $\Delta_{s} / s_{0} \gg$ 1. A loose upper (lower) bound is given by $N\left(4^{\max (K, 2 J)+1}\right)$

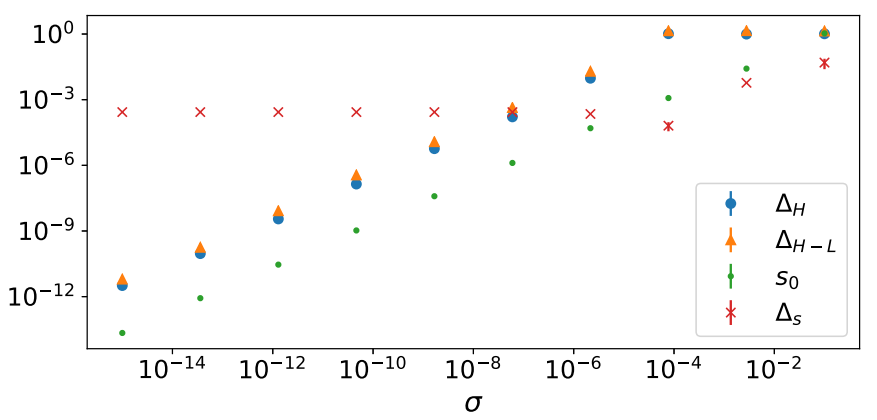

FIG. 2. $N=5$ equilibrium learning using the parameters of Fig. 1 in the presence of $\mathcal{N}(0, \sigma)$ distributed measurement noise. The estimator error grows linearly as a function of $\sigma$ and is approximately two orders of magnitude larger than the minimal singular value when the gap $\Delta_{s}$ is well defined. Results are averaged over 20 disorder configurations. 
$\left[N\left(4^{\max (K, 2 J)}\right)\right]$ measurement settings, as indicated by the dashed line labeled with the numeral "3" (“2”) in Fig. 1.

\section{STABILITY ANALYSIS}

In practice, state preparation and measurement (SPAM) errors deteriorate the estimator. Assume the correlation matrix including errors is $\tilde{C}=C+E$, where $C$ is the ideal signal and $E$ is due to SPAM perturbations. Weyl's theorem, stating that $\left|\tilde{s}_{i}-s_{i}\right| \leqslant\|E\|_{2}[13,14]$, bounds change in $s_{0}$ given $E$. For example, consider measurement noise taking $\left\langle O_{i}\right\rangle \rightarrow\left\langle O_{i}\right\rangle+$ $\delta_{O_{i}}$, where the $\left\langle O_{i}\right\rangle$ 's are matrix elements of $A$ in Eq. (3) and the open systems generalization. Here, $E$ is the collection of normally distributed, with $\mu=0$ and $\sigma^{2}$ variance, $\delta_{O_{i}}$ random variables. Figure 2 shows how, for small $\sigma, s_{0}$ and the estimator infidelity grow linearly with $\sigma$. Intuitively, $\|E\|_{2}$ goes as $\mathcal{O}(\sigma)$ and Weyl's bound's tightness is tested with 100 noise realizations in the gapped regime showing that $\|E\|_{2}=1.669(15)\left|\tilde{s}_{0}-s_{0}\right|$. The linear scaling continues until $s_{0}>\Delta_{s}$, i.e., the singular spectrum is effectively degenerate, at which point no unique solution exists.

To this end, we could also consider steady state perturbations taking $\rho_{\mathrm{SS}} \rightarrow(1-\varepsilon) \rho_{\mathrm{SS}}+\varepsilon \rho^{\prime}$ for arbitrary $\rho^{\prime}$. Due to linearity, $\left\langle O_{i}\right\rangle=(1-\varepsilon) \operatorname{Tr}\left[\rho_{\mathrm{SS}} O_{i}\right]+\varepsilon \operatorname{Tr}\left[\rho^{\prime} O_{i}\right]$, which rescales the $A \rightarrow(1-\varepsilon) A$ with the $\rho^{\prime}$ component generating the $E$ noise matrix. We have again numerically verified Weyl's bound by emulating an anisotropic depolarizing channel, i.e., $\rho^{\prime}=\sum c_{i} \hat{p}_{i} \rho_{s} s \hat{p}_{i}$, by reducing each expectation random value drawn from a folded normal distribution. Again, the standard deviation statistically determines the spectral norm and we numerically find $\|E\|_{2}=3.817(18)\left|\tilde{s}_{0}-s_{0}\right|$.

\section{DYNAMICAL LEARNING}

Lastly, we simulate the dynamical learning scenario by solving $\min _{\vec{x}}|| C^{\prime} \vec{x}-\left.\vec{W}\right|_{2} ^{2}$. References $[7,8]$ suggested product states as a dynamical basis but, as exact product states may not actually be available as a near-term resource, we consider a generalized scenario. We take the fixed-point density operator as a resource and conjugate it with respect to a set of unitary operators $\left\{U_{j}\right\}$. In this way, we generate a new state basis $\left\{\rho_{j}\right\}=\left\{U_{j} \rho_{\mathrm{SS}} U_{j}^{\dagger}\right\}$. In contrast to stationary inference any set of states will generate valid correlation matrices, given that the basis is sufficiently large, making dynamical assignment robust to state perturbations.

Choosing $\left\{U_{j}\right\}$ to consist of all 1-local Pauli operators, and beginning a time step $\delta t / c_{z} \sim 1 e-3$, we find that the finitetime protocol behaves qualitatively similarly to the steady state protocol. That is, the estimator error dramatically vanishes when the set of equations is complete with respect to the model space and then further converges as more information is collected. The normalized minimum estimator error for both the Hamiltonian and the total Hamiltonian-Lindbladian estimates is plotted in Fig. 3 as a function of system size. Here, we illustrate the errors for both components of the model system using (i) a naive least-squares fit and (ii) imposing positivity constraints on the Lindbladian process. Interestingly, Fig. 3 shows that, given the parameters considered, the Hamiltonian estimator error is insensitive to positivity constraints whereas the environmental error is greatly reduced with their inclusion.

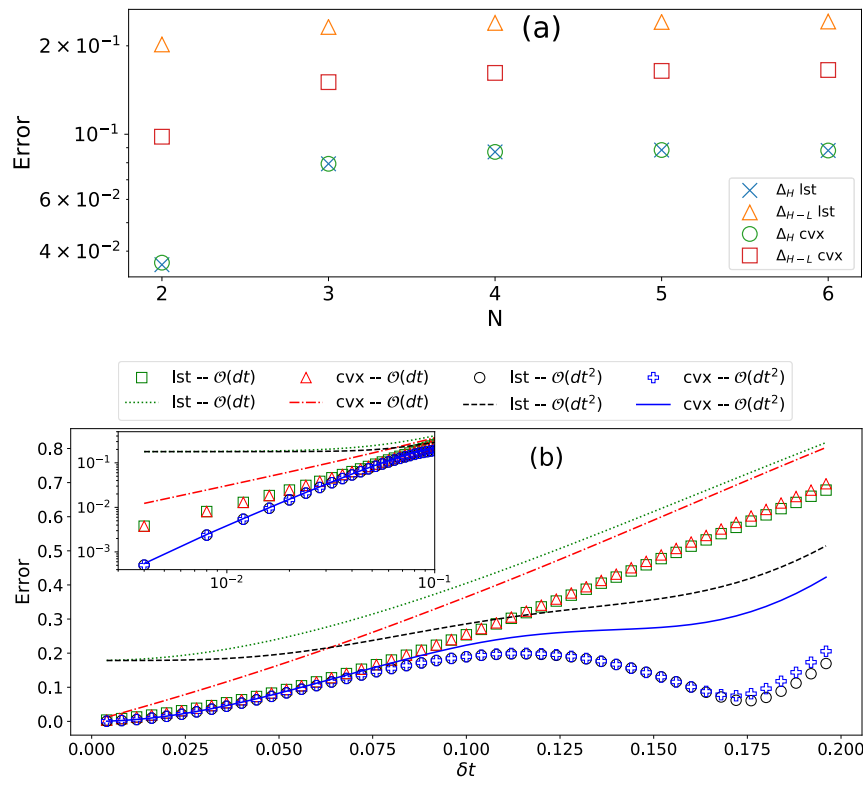

FIG. 3. Top panel: Normalized Hamiltonian and HamiltonianLindbladian reconstruction errors as a function of chain length using least-squares minimization ( $x$ 's and open circles) and minimization by convex optimization with the Lindbladian model subject to positive semidefinite constraints (open triangles and squares). We reuse the earlier model parameters and evolve for a time $\delta t / c_{z} \sim$ $1 \times 10^{-3}$. Bottom panel: Normalized reconstruction errors for firstand second-order finite-difference derivative approximations. Here, the evolution time is varied and the Hamiltonian (open symbols) and total (lines) estimator errors are given. The least-squares (lst) and convex minimization (cvx) Hamiltonians agree at small times as illustrated in the log-linear inset.

In practice, it is difficult to evolve for sufficiently small times. For example, a minimum evolution time for the OPENPULSE control framework implementation on IBM's platform [15] is $\delta t_{\min } \sim 14 \mathrm{~ns}$ (four pulse steps of $d t=32 / 9$ ). As noted in Fig. 3, high-fidelity estimators require a sampling frequency greater than the interaction frequencies, so the IBM timescale is appropriate for interactions below $\sim 100 \mathrm{MHz}$. Recent OPENPULSE updates have decreased the pulse interval by a factor of 16 which would increase the cutoff frequency to the $\mathrm{GHz}$ scale.

To ameliorate the small time requirement we employ a higher-order finite-difference approximation $[-O(2 \delta t)+$ $4 O(\delta t)-3 O(0)] / 2 \delta t=\dot{O}(0)+\mathcal{O}\left(\delta t^{2}\right)$ such that the error scales as $\mathcal{O}\left(\delta t^{3}\right)$. The bottom panel of Fig. 3 shows the Hamiltonian and composite estimator errors as a function of $\delta t$ for both the linear and quadratic finite-difference approximations. Note one major drawback of this modification lies in the increased variance for the derivative estimator. Denoting $V_{1(2)}$ as the variance in evaluating the first- (second-) order time derivative of an operator $O$, and assuming independence and that $\operatorname{Var}[O(t)]$ is constant for all $t$, we have $V_{2} / V_{1}=13 / 4$, which corresponds to an approximately tenfold increase in the number of samples to reduce the second-order estimator variance to that of the first.

Interestingly, we note that for $\delta t \gtrsim 0.15$ both the firstand second-order least-squares Hamiltonian estimator errors 
are smaller than their positive semidefinite counterparts. We attribute this counterintuitive result to the finite-difference approximation error. The least-squares minimization is afforded greater freedom in generating a nonphysical environment, which partially absorbs the finite-time error, thus estimating Hamiltonians more accurately.

\section{CONCLUSION}

In this work we have studied the task of assigning a local Hamiltonian to open quantum systems in a variety of settings. By restricting ourselves to a Lindbladian formulation, with a polynomial number of model parameters describing the evolution, we are able to generalize and implement previous local model estimation techniques in both a steady state and dynamical setting.

To validate our constructions, we have performed numerical simulations of open system Hamiltonian assignments in the context of an $X X$-interacting spin chain subject to thermal relaxation. Our results verify how in the clean limit and, contingent upon an appropriately chosen model space, Hamiltonians may be inferred both in and out of equilibrium. Furthermore, we have bounded the effects of noise and discussed simple modifications to increase the estimator accuracy in the dynamical context.

Our work paves the way for the determination of manybody Hamiltonians in open quantum systems. The extension of our results to a greater diversity of open quantum systems remains an open research direction. For example, it will be of great interest to understand how our current work can be enhanced by incorporating existing techniques such as Bayesian learning [5], and quantum process identification [16]. Note that for current superconducting devices, the measurement infidelity is $\sim 1 \%$, meaning that either these rates must fall by around two orders of magnitude or the assignment must be improved with other sources of information. It is also of great interest to determine how such methods can be applied to locally interacting non-Markovian environments and to better understand the decoupling between Hamiltonian and environmental estimation. Lastly, from an information theoretic sense, it is of interest to determine, given state resources, which sets of measurement configurations saturate Cramer-Rao bounds for Hamiltonian parameter estimation.

\section{ACKNOWLEDGMENTS}

We thank A. Seif, P. T. Bhattacharjee, and R. S. Bennink for helpful conversations and acknowledge DOE ASCR funding under the Quantum Computing Application Teams program, FWP No. ERKJ347. This research used quantum computing system resources supported by the US Department of Energy, Office of Science, Office of Advanced Scientific Computing Research program office.

This manuscript has been authored by UT-Battelle, LLC, under Contract No. DE-AC0500OR22725 with the US Department of Energy. The United States Government retains and the publisher, by accepting the article for publication, acknowledges that the United States Government retains a nonexclusive, paid-up, irrevocable, world-wide license to publish or reproduce the published form of this manuscript, or allow others to do so, for the United States Government purposes. The Department of Energy will provide public access to these results of federally sponsored research in accordance with the DOE Public Access Plan.
[1] C. M. Dawson and M. A. Nielsen, The Solovay-Kitaev algorithm, Quantum Inf. Comput. 6, 081 (2006).

[2] L. Lamata, A. Parra-Rodriguez, M. Sanz, and E. Solano, Digital-analog quantum simulations with superconducting circuits, Adv. Phys.: X 3, 519 (2018).

[3] A. Keesling, A. Omran, H. Levine, H. Bernien, H. Pichler, S. Choi, R. Samajdar, S. Schwartz, P. Silvi, S. Sachdev, P. Zoller, M. Endres, M. Greiner, V. Vuletić, and M. D. Lukin, Quantum Kibble-Zurek mechanism and critical dynamics on a programmable Rydberg simulator, Nature (London) 568, 207 (2019).

[4] Y. Wang, Quantum Computation and Quantum Information, 10th ed. (Cambridge University Press, Cambridge, UK, 2012), Vol. 27.

[5] C. E. Granade, C. Ferrie, N. Wiebe, and D. G. Cory, Robust online Hamiltonian learning, New J. Phys. 14, 103013 (2012).

[6] X.-L. Qi and D. Ranard, Determining a local Hamiltonian from a single eigenstate, Quantum 3, 159 (2017).

[7] A. Shabani, M. Mohseni, S. Lloyd, R. L. Kosut, and H. Rabitz, Estimation of many-body quantum Hamiltonians via compressive sensing, Phys. Rev. A 84, 012107 (2011).
[8] M. P. da Silva, O. Landon-Cardinal, and D. Poulin, Practical Characterization of Quantum Devices without Tomography, Phys. Rev. Lett. 107, 210404 (2011).

[9] E. Bairey, I. Arad, and N. H. Lindner, Learning a Local Hamiltonian from Local Measurements, Phys. Rev. Lett. 122, 020504 (2019).

[10] H. P. Breuer and F. Petruccione, The Theory of Open Quantum Systems (Oxford University Press, Oxford, UK, 2007).

[11] E. Bairey, C. Guo, D. Poletti, N. H. Lindner, and I. Arad, Learning the dynamics of open quantum systems from local measurements, New J. Phys. 22, 032001 (2020).

[12] J. R. Johansson, P. D. Nation, and F. Nori, QuTiP 2: A Python framework for the dynamics of open quantum systems, Comput. Phys. Commun. 184, 1234 (2013).

[13] H. Weyl, Das asymptotische Verteilungsgesetz der Eigenwerte linearer partieller Differentialgleichungen (mit einer Anwendung auf die Theorie der Hohlraumstrahlung), Math. Ann. 71, 441 (1912).

[14] G. W. Stewart, Perturbation theory for the singular value decomposition, in SVD and Signal Processing, II: 
Algorithms, Analysis and Applications (Elsevier, Amsterdam, 1990), pp. 99-109.

[15] D. C. McKay, T. Alexander, L. Bello, M. J. Biercuk, L. Bishop, J. Chen, J. M. Chow, A. D. Córcoles, D. Egger, S. Filipp, J. Gomez, M. Hush, A. Javadi-Abhari, D. Moreda, P. Nation, B. Paulovicks, E. Winston, C. J. Wood, J. Wootton, and J. M.
Gambetta, Qiskit backend specifications for OpenQASM and OpenPulse experiments, arXiv:1809.03452.

[16] R. S. Bennink and P. Lougovski, Quantum process identification: a method for characterizing non-Markovian quantum dynamics, New J. Phys. 21, 083013 (2019). 